\title{
Synthesis and characterization of novel polyimides with diamines containing thiophene moieties
}

\author{
Mu-Ju Yoon and Tae-Ho Yoon * \\ School of Materials Science and Engineering, Gwangju Institute of Science and Technology (GIST) \\ 1 Oryong-dong, Buk-gu, Gwangju 500-712, Republic of Korea
}

\begin{abstract}
Noble diamine monomers containing thiophene moiety were prepared, such as 2,5"'-diamino-2, $2^{\prime}: 5^{\prime}, 2^{\prime \prime}: 5^{\prime \prime}, 2^{\prime \prime \prime}$-quaterthiophene (4TDA) and 2, $5^{\prime \prime \prime \prime \prime}$ -diamino-3", $4^{\prime \prime \prime}-$ dihexyl-2,2':5', $2^{\prime \prime}: 5^{\prime \prime}, 2^{\prime \prime \prime}: 5^{\prime \prime \prime}, 2^{\prime \prime \prime \prime}: 5^{\prime \prime \prime \prime}$, $2^{\prime \prime \prime \prime \prime}$-sexithiophene (6TDA). Then, these monomers were utilized to prepare polyimides with 3 ,6-diphenylpromellitic dianhydride (DPPMDA), 3,6-di(4'-trifluoro-methylphenyl) pyromellitic dianhydride (6FPMDA) or 3,6-di(3',5'-bis (trifluoromethyl)phenyl) pyromellitic dianhydride (12FPMDA) via a conventional two-step process. The polyimides were characterized by FT-IR, TGA and DSC, and then subjected CV, UV-vis and PL measurements. The polyimides containing thiophene moiety exhibited high glass transition temperatures $\left(280 \sim 310^{\circ} \mathrm{C}\right)$ and excellent thermal stability $\left(>420^{\circ} \mathrm{C}\right)$ in air as well as green emission $(535 \sim 586 \mathrm{~nm})$
\end{abstract}

During past decades, conjugated polymers have been investigated for optoelectronic applications due to their light weight, flexibility and possible inexpensive solution processing [1]. Thus, a number of conjugated polymers based on thiophene, phenylenevinylene, fluorine, carbazole and triphenylamine have been prepared. However, these polymers exhibited relatively poor optical and/or electrical properties, as well as very poor durability [2] which is being attributed to their low Tgs.

Thus, there has been a great amount of research on increasing the $\mathrm{Tg}$ of conjugated polymers via copolymerization [3], incorporation of bulky side groups [4], or preparation of ladder-type [5]. Unfortunately, however, these approaches exhibited only $\sim 200{ }^{\circ} \mathrm{C}$ [4]. Consequently, incorporation of imide moiety was suggested owing to its very high $\mathrm{T}_{\mathrm{g}}\left(>300{ }^{\circ} \mathrm{C}\right)$ [6], leading to polyimide copolymers with conjugated groups [7-9] or homo-polyimide with conjugated side moiety [10]. Some of these resulted in high $\mathrm{T}_{\mathrm{gS}}\left(\sim 300{ }^{\circ} \mathrm{C}\right)[8,10]$ but their optical and electrical properties are not so good.

*To whom correspondence should be addressed. E-mail: thyoon@gist.ac.kr

Received April 17. 2012. Accepted May 18. 2012
In this study, therefore, it was attempted to prepare thiophene-based diamine monomers such as 2,5'"-diamino-2,2':5',2":5",2'"-quaterthiophene (4TDA) and 2,5"'"'-diamino-3",4"'-dihexyl-2,2':5',2":5",2"':5'"',2"'":5"'",2"'" '-sexi thiophene (6TDA). Then, these monomers were utilized to prepare polyimides with PMDA-based dianhydrides containing electron withdrawing fluorine groups.

Scheme 1. Synthesis of 6TDA

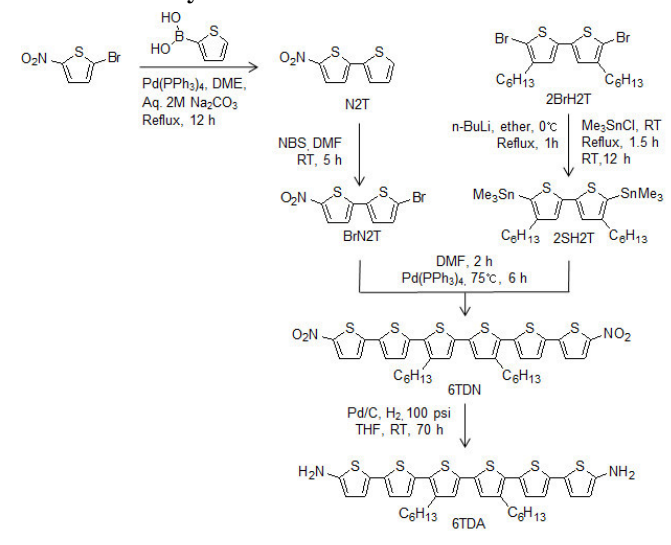

The 6TDA was synthesized via 7-step process (Scheme 1). First, 5-bromo-5'-nitro-2,2'-bithiophene (BrN2T) was obtained by bromination of 5-nitro-2, 2'-bithiophene (N2T) which was prepared via Suzuki coupling reaction from 2-thiopheneboronic acid and 2-bromo-5-nitrothiophene [12]. Next, this was reacted with 2,5-bis (trimethylstannyl)-4,4'-dihexyl-2,2'-bithiophene (2SH2T) which was prepared from 2,5'-dibromo-4, 4'-dihexyl-2,2'-bithiophene (2BrH2T) [13] via lithiation using $n \mathrm{BuLi}$ and then reacting with trimethyltin chloride, resulting in 2,5"'"'-dinitro-3", 4 "'-dihexyl-2,2':5',2":5",2"':5"', $2^{\prime \prime \prime}: 5 " ', 22^{\prime \prime \prime '-s e x i t h i o p h e n e ~(6 T D N) ~ w h i c h ~ w a s ~ h y d r o g e n a t e d ~}$ to afford 6TDA (see Supporting information for detailed synthesis). The product was purified by column chromatography using methylene chloride, providing redish oil (yield 11.9\%). ${ }^{1} \mathrm{H}-\mathrm{NMR}$ (DMSO, ppm): 7.21 $(\mathrm{s}, 2 \mathrm{H}), 7.05(\mathrm{~d}, 2 \mathrm{H}), 6.91(\mathrm{~d}, 2 \mathrm{H}), 6.84(\mathrm{~d}, 2 \mathrm{H}), 5.97$ (s, 4H), $5.83(\mathrm{~d}, 2 \mathrm{H}), 2.71(\mathrm{t}, 4 \mathrm{H}), 1.62(\mathrm{t}, 4 \mathrm{H})$, $1.35 \sim 1.30(\mathrm{~m}, 12 \mathrm{H})$ and $0.86(\mathrm{t}, 6 \mathrm{H})$. FT-IR $(\mathrm{KBr}$, $\mathrm{cm}^{-1}$ ): 3380, 3280 (primary amines stretch), 3100 2920 (aryl-H stretching), 2950 2750 (alkyl C-H stretch), 
1690 1560 (N-H bending), 990 (C-N stretch) and 550 (N-H out-of-plane bending).

For comparison, 4TDA was also synthesized via similar chemistry from 5, 5-bis (trimethylstannyl)-2, 2' -bithiophene (2S2T) and 2-bromo-5-nitrothiophene via Stille coupling reaction, resulting in 2,5'"-dinitro-2,2':5',2":5", 2 "'-quaterthiophene (4TDN) which was then hydrogenated and purified by recrystallization using chloroform, affording greenish crystals (yield 44.5\%). ${ }^{1} \mathrm{H}-\mathrm{NMR}$ (DMSO, ppm): $7.05(\mathrm{~d}, 2 \mathrm{H}), 6.82 \sim 6.77(\mathrm{~m}, 4 \mathrm{H}), 5.99(\mathrm{~s}, 2 \mathrm{H})$ and 5.81 (d, 2H). FT-IR $\left(\mathrm{KBr}, \mathrm{cm}^{-1}\right): 3380,3280$ (primary amines stretch), 1690 1560 (N-H bending), 990 (C-N stretch) and 550 (N-H out-of-plane bending).

Scheme 2. Synthesis of polyimdie with 6TDA

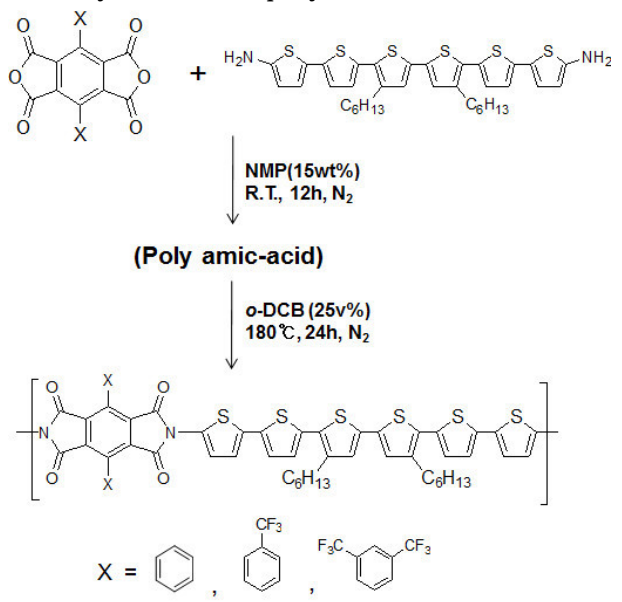

These monomers were utilized to synthesize polyimides with dianhydrides such as 3,6-di (3',5'-bis (trifluoromethyl) phenyl pyromellitic dianhydride (12FPMDA) [13], 3,6-di (4'-trifluoromethylphenyl) pyromellitic dianhydride (6FPMDA) [14] or 3,6-diphenyl pyromellitic dianhydride (DPPMDA) [15], sd shown in Scheem2. The polyimides were prepared by two-step process; synthesis of poly (amic-acid), followed by solution imidization [13]. The polymerization was conducted in NMP at $15 \mathrm{w} / \mathrm{v} \%$ solid concentration and the number-average molecular weight was controlled to $25,000 \mathrm{~g} / \mathrm{mole}$ by off-stoichiometry. The solution imidization was performed in the presence of o-DCB (20 v.\%) at $180{ }^{\circ} \mathrm{C}$ for $24 \mathrm{hr}$ under a nitrogen flow. Upon completion of polymerization, the polymer was isolated by precipitating into methanol and analyzed by FT-IR (KBr), TGA and DSC.

The hiophene-based polyimide, for example 12FPMDA6TDA, exhibited asymmetric $\left(1760 \mathrm{~cm}^{-1}\right)$ and symmetric (1710 $\left.\mathrm{cm}^{-1}\right) \quad \mathrm{C}=\mathrm{O}$ stretching and C-N stretching (1315 $\left.\mathrm{cm}^{-1}\right)$, but no amide-carbonyl $(\mathrm{C}=\mathrm{O})$ peak at $1650 \mathrm{~cm}^{-1}$, indicating successful imidization. In addition, $\mathrm{CF}_{3}$ stretching-bending absorption $\left(1120 \mathrm{~cm}^{-1}\right)$ was also observed, demonstrating presence of fluorine moieties [13] . This polyimide also showed good solubility in NMP, DMF TCE, THF and $\mathrm{CHCl}_{3}$, but slightly poorer solubility was observed with 6FPMDA and DPPDMA (See supporting information)

The polyimide of DPPMDA-6TDA exhibited very high $T_{g S}$ of $310^{\circ} \mathrm{C}$, followed by 299 with 6FPMDA and $291^{\circ} \mathrm{C}$ with $12 \mathrm{FPPMDA}$, while those with 4TDA showed 303, 291 and $281{ }^{\circ} \mathrm{C}$ demonstrating the effect of $\mathrm{CF}_{3}$ moiety, as expected[13], as well as thiophene moiety. These polyimide also exhibited excellent thermal stability (> $420{ }^{\circ} \mathrm{C}, 5 \mathrm{wt} \%$ loss temperature) in air in TGA analysis. The best value was obtained with DPPMDA-4TDA $\left(454^{\circ} \mathrm{C}\right)$, demonstrating again the effect of $\mathrm{CF}_{3}$ and thiophene units.
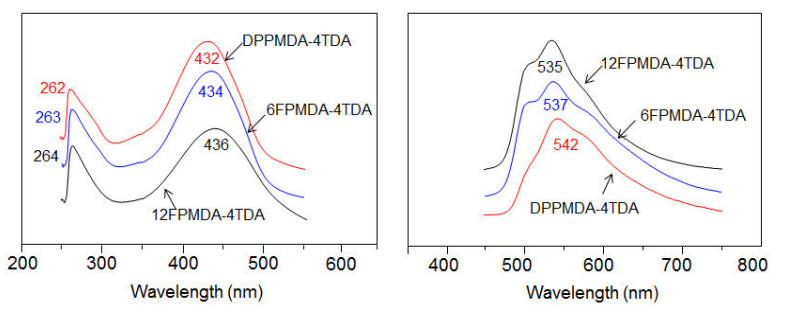

Figure 1. Absorption (left) and PL emission (right) of polyimides with 4TDA.

These polyimides exhibited UV-vis absorptions at around $430 \mathrm{~nm}$ and $440 \mathrm{~nm}$ with 4TDA and 6TDA, respectively (Table 1), while their PL emissions appeared at 530-540 $\mathrm{nm}$ and 550-580 nm, respectively. As noted, 4TDA-based polyimide provided shorter wavelength PL emissions compared to those with 6TDA, possibly attributing to destruction molecular packing because of the hydrocarbon attached to 6TDA, while blue shift in PL emission with 6FPMDA and 12FPMDA can be attributed electron withdrawing nature of $\mathrm{CF}_{3}$ moiety.

Table 1. Optical and electrical properties of polyimides with thiophene moiety.

\begin{tabular}{|c|c|c|c|c|c|c|}
\hline \multicolumn{2}{|c|}{ Polyimide } & $\begin{array}{c}\mathrm{Abs} \lambda_{\text {abs }} \\
(\mathrm{nm})^{\mathrm{a}}\end{array}$ & $\begin{array}{c}\mathrm{PL} \lambda_{\text {emi }} \\
(\mathrm{nm})^{\mathrm{b}}\end{array}$ & $\begin{array}{c}\mathrm{HOMO} \\
(\mathrm{eV})^{\mathrm{c}}\end{array}$ & $\begin{array}{c}\text { LUMO } \\
(\mathrm{eV})^{\mathrm{d}}\end{array}$ & $\begin{array}{c}\text { Band gap } \\
(\mathrm{eV})\end{array}$ \\
\hline \multirow{4}{*}{4 4TDA } & DPPMDA & 262,432 & 542 & 5.38 & 2.44 & 2.94 \\
\cline { 2 - 7 } & $6 F P M D A$ & 263,434 & 537 & 5.42 & 2.34 & 3.08 \\
\cline { 2 - 8 } & $12 F P M D A$ & 264,436 & 535 & 5.75 & 2.41 & 3.34 \\
\hline \multirow{4}{*}{6 6TDA } & DPPMDA & 264,444 & 586 & 5.34 & 2.42 & 2.92 \\
\cline { 2 - 8 } & 6FPMDA & 265,447 & 568 & 5.38 & 2.33 & 3.05 \\
\cline { 2 - 8 } & 12FPMDA & 267,450 & 554 & 5.68 & 2.30 & 3.34 \\
\hline
\end{tabular}

From the cyclic voltammetry (CV) of these polyimides, onsets of oxidation potentials were measured at 0.54, 0.58 and $0.84 \mathrm{~V}$ with DPPMDA-6TDA, 6FPMDA-6TDA and 12FPMDA-6TDA (Table 1), respectively, while slightly higher potential was observed with DPPMDA-4TDA(0.58), 6FPMDA-4TDA (0.62) and 12FPMDA-4TDA(0.95 V). From these values, HOMO level energies were calculated [16], and then LUMO energies were estimated with optical bandgap estimated from UV-vis absorption edge values (Table 1). These values are compatible to those in the literature [9]. 
In summary, noble dimaine monomers, containing thiophene moiety, such as 4TDA and 6TDA were successfully prepared and then utilized to synthesized polyimides with DPPMDA, 6FPMDA or 12FPMDA. The polyimides exhibited very high $\operatorname{Tg}\left(281 \sim 310^{\circ} \mathrm{C}\right)$ and excellent thermal stability (5wt\% loss temperature of $>420^{\circ}$ C) and green emission (535 586nm).
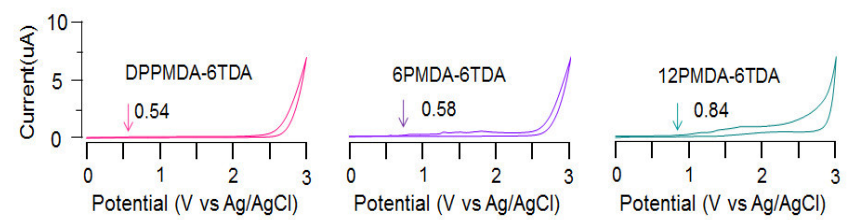

Figure 2. Cyclic voltammetry of polyimides with thiophene moiety.

Received: April 16, 2012

Revised: May 18, 2012.

Published online: June 15, 2012

KEYWORDS: thiophene moiety, polymide, high Tg, excellent thermal stability, green emission

\section{ACKNOWLEDGEMENT}

This work was supported by the National Research Foundation of Korea (Mid-career Researcher Program) (No.2007-0056941) and BK21 program.

\section{SUPPORTING INFORMATION}

Experimental procedures; synthetic and characterization of the imide compounds are available free of charge via the Internet at http://rcp.or.kr

\section{REFERENCES AND NOTES}

1. Gunes, S.; Neugebauer, H.; Sariciftci, N. S. Chem. Rev. 2007, 107, 1324-1338.

2. Bernius, M.T.; Inbasekaran, M.; O’ Brien, J.; Wu, W. Adv. Mater. 2000, 12, 1737-1750.

3. Park, J. W.; Park, S. J.; Kim, Y.H.; Shin, D. C.; You, H.; Kwon, S. K. Polymer 2009, 50, 102-106.

4. Pogantsch, A.; Wenzl, F. P.; List, E. J. W.; Leising, G.; Grimsdale, A.C.; MDllen, K. Adv. Mater. 2002, 14, 1061-1064.

5. Mishra, A.K.; Graf, M.; Grasse, F.; Jacob, J.; List, E.J.W.; M Mllen, K. Chem. Mater. 2006, 18, 28792885.

6. Ng, W. Y.; Gong, X.; Chan, W. K. Chem. Mater. 1999, 11, 1165-1170.

7. Kotov, B. V.; Rumyantsev, B. M.; Berendyaev, V. I.; Lunina, E. V.; Bespalov, B. P.; Frankevich, E. L.; Triebel, M. M. Synth. Met. 2001, 121, 1553-1554.

8. Jung, M. S.; Lee, T. W.; Lee, J. H.; Shon, B. H.; Jung, I. S.; Polymer 2006, 47, 2670-2676.

9. Hsiao, S. H.; Liou, G. S.; Kung, Y. C.; Lee, Y. J.; Eur. Polym. J. 2010, 46, 1355-1366.
10. Choi, J .K.; Cho, K.; Yoon, T. H. Synth. Met. 2010, 160, 1938-1944.

11. Takahashi, M.; Masui, K.; Sekiguchi, H.; Kobayashi, N.; Mori, A.; Funahashi M.; Tamaoki, N. J. Am. Chem. Soc. 2006, 128, 10930-10933

12. Dingemans, T. J.; Murthy N. S.; Samulski, E. T. J. Phys. Chem. B. 2001, 105, 8845-8860.

13. Myung, B. Y.; Kim J. J.; Yoon, T. H. J. Polym. Sci., Part A: Poly. Chem. 2002, 40(23), 4217-4227.

14. Harris, F. W.; Lin, S. H.; Li, F.; Cheng, S. Z. D. Polymer 1996, 37, 5049-5057.

15. Tomikawa, M.; Cheng S. Z. D.; Harris, F.W. Polym. Preprint. 1995, 36, 707-708

16. Sensfuss, S.; Al-Ibrahim, M.; Organic Photovoltaics: Mechanism, Materials and Devices, CRC Press, Boca Raton, FL, 2005, p.534. 